\title{
Performance Analysis of Proactive, Reactive and Hybrid MANET Routing Protocols on IEEE 802.11 Standard
}

\author{
Anjali \\ Dept. of Computer Science \\ Punjabi University, Patiala
}

\author{
Maninder Singh \\ Dept. Of Computer Science \\ Punjabi University, Patiala
}

\begin{abstract}
MANET consists of mobile nodes which exchange information dynamically among them over wireless links. The most important element of MANET is Routing Protocols which are needed to handle dynamic communication and also find route so as to deliver data packets to the correct destination. Performance of routing protocols is an important issue because of dynamic nature of MANET. In this paper performance of AODV, OLSR and GRP routing protocols is evaluated for FTP based application traffic on IEEE 802.11 WLAN Standard and 48 Mbps data rate. The network performance is evaluated by using OPNET simulator based on various quantitative metrics- Network Load, Throughput, Retransmission Attempts and Media Access Delay by varying physical characteristics and number of nodes. A comparative performance analysis of these protocols have been carried out in this paper and in the last conclusion will be presented which demonstrate that performance of routing protocols differs by varying the network and selection of accurate routing protocol according to the network ultimately influences the efficiency of the network in a magnificent way.
\end{abstract}

\section{Keywords}

AODV, GRP, IEEE 802.11, MANET, OLSR, OPNET, Routing protocols

\section{INTRODUCTION}

Mobile Ad hoc Network (MANET) is a group of mobile nodes which communicate with each other without any centralized support and are connected by wireless links. Each node in MANET acts as a router or an end system for all other nodes in the network. Mobile ad hoc network supports competent operation by integrating routing in nodes of mobile ad hoc wireless networks.

During data communications, performance of MANET routing protocols is a significant issue. Therefore, effective and accurate routing protocols are required so as to handle mobility of nodes and to give superior utilization in MANET technology. An ad hoc routing protocol is a standard that handles how nodes determine which way to forward packets between devices in a mobile ad-hoc network. In this paper we have evaluated performance of AODV, OLSR AND GRP routing protocols by considering FTP application type and IEEE 802.11a/g WLAN Standard.

IEEE 802.11 is a WLAN Standard with data rates up to 2 Mbps which is developed in 1997.After that, various task groups have been developed to extend this standard like 802.11a, 802.11b and 802.11g.
The 802.11a task group created a standard for WLAN operation in the $5 \mathrm{GHz}$ band, with data rates up to $54 \mathrm{Mbps}$.

The 802.11b task group produced a standard for WLAN operations in $2.4 \mathrm{GHz}$ band, with data rates up to $11 \mathrm{Mbps}$ and backward compatibility. It published in $1999 . \mathbf{8 0 2 . 1 1 g}$ is working to develop a higher speed extension to the $802.11 \mathrm{~b}$. The IEEE 802.11standard defines two operational modes for WLANs: infrastructure-based and infrastructure-less or ad hoc. Network interface cards can be set to work in either of these modes but not in both simultaneously [1]

\section{RELATED WORK}

Razan Al-Ani [2] simulated and evaluated the AODV, OLSR, GRP, TORA and DSR routing protocols to analysis the performance on basis of Throughput, Delay, and Network load. They created a network which consists of mobile nodes, one fixed WLAN server running GRP and RX group config node to speed up simulation time. All nodes are configured to work with 5.5 Mbps data rate and FTP application type was chosen for all nodes with multiple FTP sessions. They ran four scenarios for each type of routing protocol. Each scenario was run for $30 \mathrm{~min}$. According to their results OLSR routing protocol performs better than others in both delay and throughput.

Xiaoyan Hong [3] surveys the routing protocols that address scalability. The routing protocols they intend to include in the survey fall into three categories: flat routing protocols, hierarchical routing approaches, and GPS augmented geographical routing schemes. In this paper, descriptions of the protocols have been provided and the differences among them have been discussed, highlighting particular important features impacting scalability. No protocol emerges as the winner for all the scenarios. They concluded that protocols have different, competitive and complementary advantages and are thus appropriate for different applications.

Ravinder Ahuja [4] evaluated performance of three types of routing protocols (AODV, OLSR and ZRP) based on random waypoint mobility model. In this paper they analyze and compare the performance of protocols using Qualnet 4.5 from scalable network .These routing protocols were compared in terms of Packet delivery ratio, Average end-to end delay and Throughput when subjected to change in no. of nodes and pause time. Simulation results show that Reactive protocols better in terms of packet delivery ratio and throughput

Kuldeep vats [5] analyzed the performance of DSR, OLSR and GRP routing protocols. They used OPNET simulation tool. They created a network containing 150 mobile nodes with the data rate of $18 \mathrm{mbps}$ and transmit power of 0.11 watts. Each node moves randomly within the network range 10,000 sq m and Simulation time was $1000 \mathrm{sec}$. According to 
their simulation result OLSR presented the best performance and GRP presented low to OLSR and high to DSR or finally DSR presented the low performance (DSR $<$ GRP $<$ OLSR) is analyzed.

\section{MOBILE Ad hoc NETWORK ROUTING PROTOCOLS}

A number of routing protocols have been created and implemented for MANET which are categorized into three different types according to their functionality: Proactive, Reactive and Hybrid.

\subsection{Proactive Protocols}

Proactive methods are also called Table-driven methods which keep routes to all other nodes in the network also considering those nodes to which packets are not sent. Thus, the route is already available when a path to a particular destination is needed at a node and there is no extra delay due to route discovery. OLSR is an example of Proactive Protocol

\subsubsection{Optimized Link State Routing Protocol (OLSR) Routing Protocol:}

OLSR is a table driven or Proactive, link-state routing protocol. Link-state routing algorithms choose best route by determining various characteristics like link load, delay, bandwidth etc. Link-state routes are more stable, accurate and reliable in calculating best route. Conceptually, OLSR contain three generic elements: a mechanism for neighbour sensing, a mechanism for efficient flooding of control traffic, and a specification of how to select and diffuse sufficient topological information in the network in order to prove optimal routes [6][ 7].

\subsection{Reactive Protocols}

Reactive methods (On-demand methods) are bandwidth efficient. Routes between nodes are determined whenever a path is required by a node to forward packets. Therefore, routing overhead is decreased because search for the route is not required on which packet is not sent. AODV is an example of Reactive Protocol.

\subsubsection{Ad hoc On-Demand Distance Vector (AODV) Routing Protocol:}

AODV [8] algorithm enables dynamic, self-starting, multi hop routing between participating mobile nodes wishing to establish and maintain an ad hoc network. AODV allows mobile nodes to obtain routes quickly for new destinations, and does not require nodes to maintain routes to destinations that are not in active communication. Route Requests (RREQs), Route Replies (RREPs) and Route Errors (RERRs) are message types defined by AODV [8].

\subsection{Hybrid Protocols}

It combine characteristics of both pro-active and re-active routing in order to find effective and reliable routes, without large control overhead, by locally using pro-active routing and inter-locally using re-active routing. In this method communication in MANET is possible when nodes are near to each other and the supposition that changes in topology are only important if they happen in the vicinity of a node. This method decides its strategies of routing according to characteristics of network and thus provides a good method for routing in MANETs. GRP is an example of Hybrid Protocol.

\subsubsection{Gathering-based Routing Protocol (GRP):}

Gathering-based Routing Protocol (GRP) gathers network information rapidly at a source node without spending a large amount of overheads. It offers an efficient framework that can simultaneously draw on the strengths of Proactive routing protocol (PRP) and reactive routing protocol (RRP) collects network information at a source node at an expense of a small amount of control overheads. The source node can equip promising routes on the basis of the collected information, thereby continuously transmitting data packets even if the current route is disconnected, its results in achieving fast (packet) transfer delay without unduly compromising on (control) overhead performance[9]

\section{EXPERIMENTAL SET-UP}

In this paper the simulation tool used for analysis is OPNET. The simulation parameters are summarized in following tables.

TABLE 1 Simulation Parameters

\begin{tabular}{|l|l|}
\hline Simulation Parameter & Value \\
\hline Simulator & OPNET Modeler 14.5 \\
\hline Area & $1000 * 1000(\mathrm{~m})$ \\
\hline Network Size & 75 and 150 nodes \\
\hline Data Rate & $48 \mathrm{Mbps}$ \\
\hline Mobility Model & Random way point \\
\hline Traffic Type & FTP \\
\hline Simulation Time & 400 sec \\
\hline Address Mode & IPV4 \\
\hline Standard & IEEE 802.11 \\
\hline Routing Protocols & AODV, OLSR, GRP \\
\hline
\end{tabular}

TABLE 2 AODV Parameters

\begin{tabular}{|l|l|}
\hline Attribute & Value \\
\hline Hello Interval(Sec) & Uniform(1,1.1) \\
\hline Allowed Hello Loss & 4 \\
\hline Net Diameter & 30 \\
\hline Node Traversal Time(Sec) & 0.06 \\
\hline $\begin{array}{l}\text { Route Error Rate } \\
\text { Limit(pkts/sec) }\end{array}$ & 12 \\
\hline Timeout Buffer & 3 \\
\hline TTL Increment & 4 \\
\hline TTL Threshold & 8 \\
\hline Local Add TTL & 4 \\
\hline Packet Queue Size(Packets) & Infinity \\
\hline Local Repair & Enabled \\
\hline Addressing Mode & IPV4 \\
\hline
\end{tabular}

TABLE 3 GRP Parameters

\begin{tabular}{|l|l|}
\hline Attribute & Value \\
\hline Hello Interval(Sec) & Uniform(4.0,5.4) \\
\hline $\begin{array}{l}\text { Neighbor Expiry } \\
\text { Time(Sec) }\end{array}$ & Constant(30) \\
\hline Distance Moved(Meters) & 2000 \\
\hline $\begin{array}{l}\text { Position Request } \\
\text { Timer(Sec) }\end{array}$ & 10.0 \\
\hline Backtrack Option & Enabled \\
\hline Routes Export & Enabled \\
\hline Number Of Initial Floods & 2 \\
\hline
\end{tabular}


Table 4 OLSR Parameters

\begin{tabular}{|l|l|}
\hline Attribute & Value \\
\hline Willingness & $\begin{array}{l}\text { Willingness } \\
\text { Default }\end{array}$ \\
\hline Hello Interval(Sec) & 2.0 \\
\hline TC Interval(Sec) & 5.0 \\
\hline Neighbor Hold Time(Sec) & 6.0 \\
\hline Topology Hold Time(Sec) & 15.0 \\
\hline $\begin{array}{l}\text { Duplicate Message Hold } \\
\text { Time(Sec) }\end{array}$ & 30.0 \\
\hline Addressing Mode & IPV4 \\
\hline
\end{tabular}

Table 5 Wireless LAN Parameters

\begin{tabular}{|l|l|}
\hline Attribute & Value \\
\hline Physical Characteristics & $\begin{array}{l}\text { Extended Rate } \\
\text { PHY(802.11g) and } \\
\text { OFDM(802.11a) }\end{array}$ \\
\hline Data Rate & $48 \mathrm{Mbps}$ \\
\hline Short Retry Limit & 9 \\
\hline Long Retry Limit & 7 \\
\hline $\begin{array}{l}\text { Max Receive } \\
\text { Lifetime(Sec) }\end{array}$ & 1.5 \\
\hline Buffer Size(bits) & 1024000 \\
\hline Roaming Capability & Enabled \\
\hline
\end{tabular}

Fig. 1 shows the simulation environment of one scenario containing 75 WLAN mobile nodes, one fixed WLAN Server, Application definition, Profile definition and Mobility Config. We configure the nodes in the scenario to work with $48 \mathrm{Mbps}$. The network size is $1000 * 1000$ meters. After that IPV4 addressing is assigned to all nodes. The "Application Config" node is used to specify applications using available application types. FTP application type was chosen to all nodes in the network with multiple FTP sessions, and the FTP was selected as high traffic load. We configured the profile with FTP application. Random waypoint mobility model was used in this simulation. Mobile nodes move at a constant speed of $10 \mathrm{~m} / \mathrm{s}$, and when reaches the destination, the pause time is $120 \mathrm{sec}$ and after that it choose a new random destination. We ran four scenarios for each type of routing protocol (AODV, OLSR, and GRP). Two scenarios ran for IEEE 802.11a WLAN Standard, one for 75 nodes and other for 150 nodes and similarly two scenarios for IEEE 802.11g WLAN Standard. Each scenario was run for 400 seconds. After successful completion of the simulations, results are selected according to the problem solution. Results are collected in the form of graphs, with overlaid data displaying multiple values.

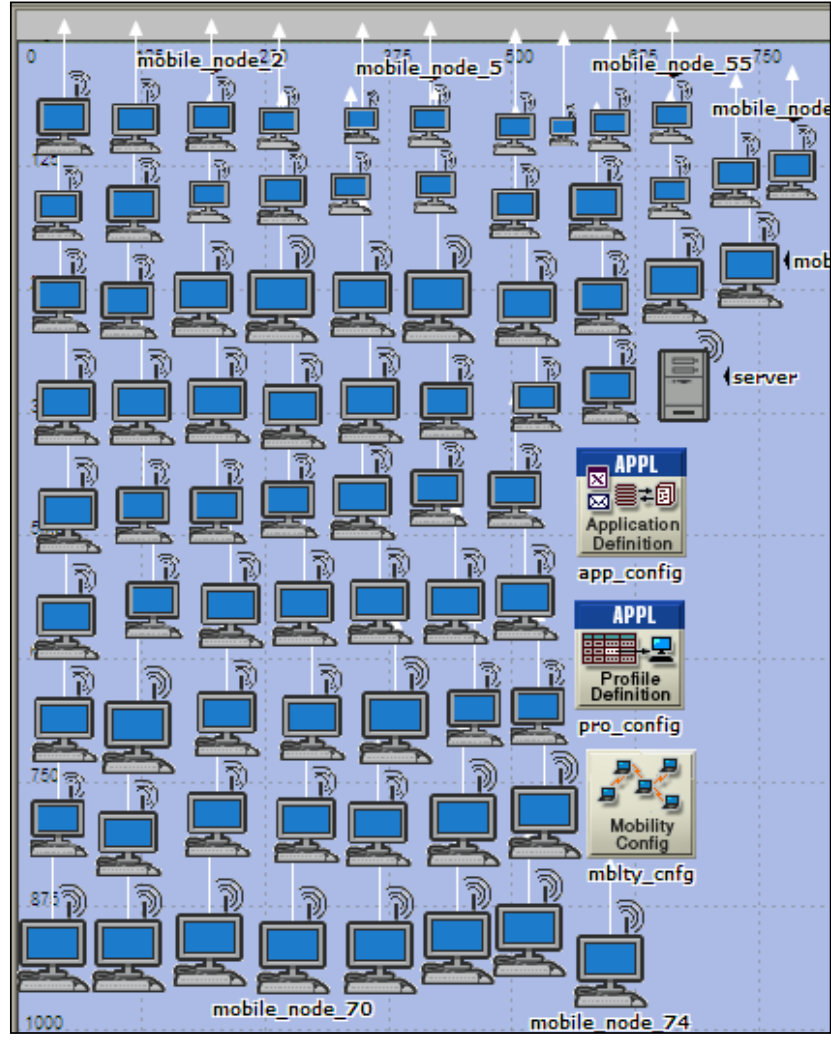

Fig 1: Network Model (75 Nodes)

\section{PERFORMANCE MERICS 5.1 Network Load}

It represents the total load (in bits/sec) submitted to wireless LAN layers by all higher layers in all WLAN nodes of the network.

\subsection{Throughput}

It represents the total number of bits (in bits/sec) forwarded from wireless LAN layers to higher layers in all WLAN nodes of the network.

\subsection{Retransmission Attempts}

It is the total number of retransmission attempts by all WLAN MACs in the network until either packet is successfully transmitted or it is discarded as a result of reaching short or long retry limit.

\subsection{Media Access Delay}

It represents the global statistic for the total of queuing and contention delays of the data, management, delayed BlockACK and Block-ACK Request frames transmitted by all WLAN MACs in the network. 


\section{SIMULATION RESULTS AND ANALYSIS}

\subsection{AODV, OLSR and GRP in 75 nodes for 802.11a and 802.11g WLAN Standard}

Figure (2 - 9) below show media access delay, network load, retransmission attempts and throughput in 75 mobile nodes scenario for 802.11a and g standard at $48 \mathrm{Mbps}$ data rate with AODV, GRP and OLSR respectively. The color scheme is showing the protocols behavior in different graphs which gives the average values. Blue line gave the results for AODV protocol, red line is for GRP and green line provide the result for OLSR routing protocol. From these average values we will conclude the behavior of all these routing protocols.

\subsubsection{Media Access Delay}

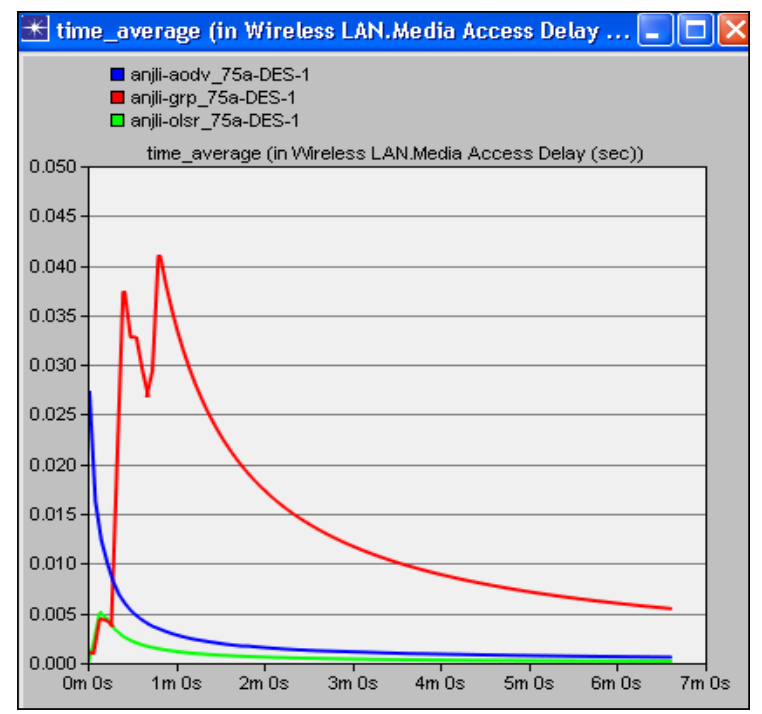

Fig 2: Media Access Delay for 802.11a with 75 nodes

According to simulation, as we can see in Fig. 2, media access delay in GRP is highest and the minimum value of media access delay belongs to OLSR.

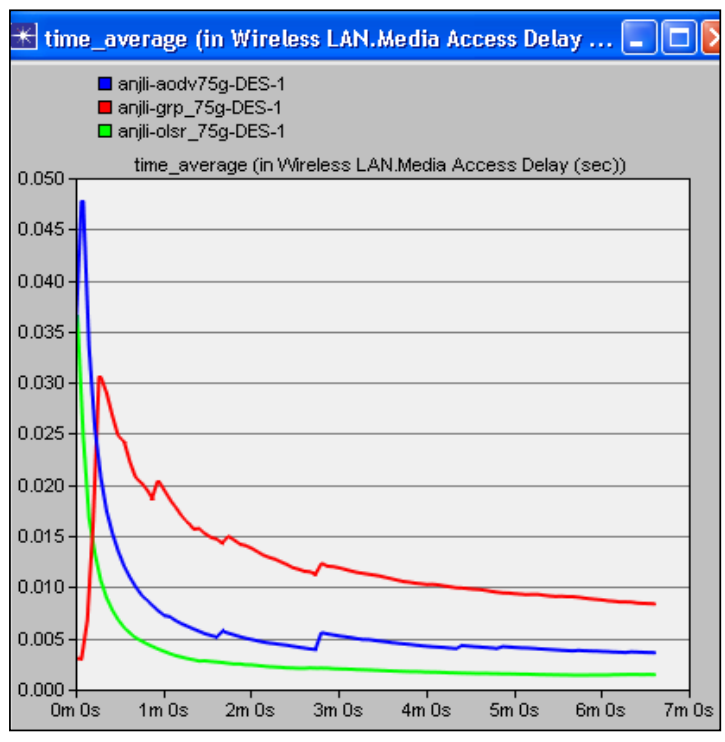

Fig 3: Media Access Delay for 802.11g with 75 nodes
The Fig. 3 show media access delay present while using 802.11g. According to this, the GRP has highest and OLSR has the minimum media access delay.

\subsubsection{Network Load}

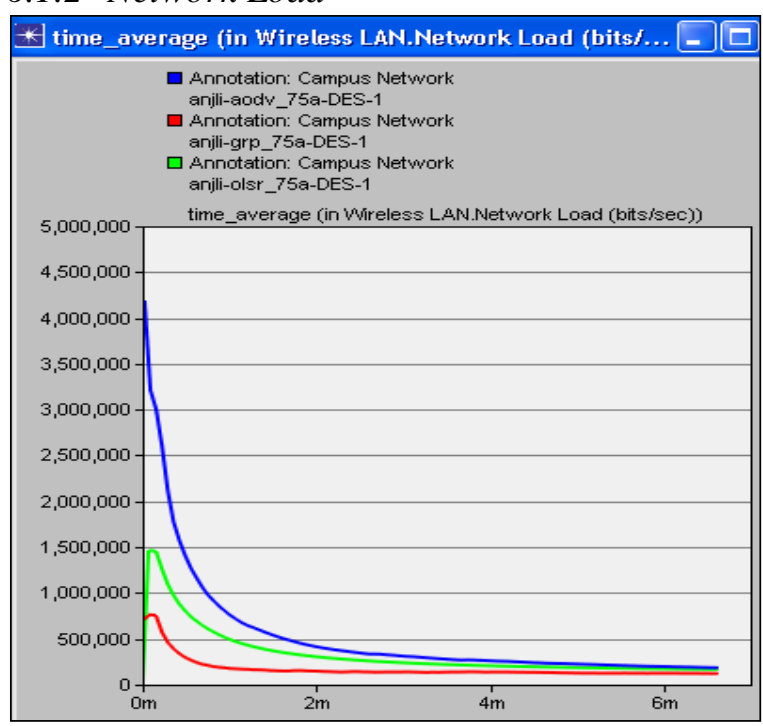

Fig. 4: Network Load for 802.11a with 75 nodes

In Fig. 4, we see that AODV has highest network load than GRP and OLSR at the start of simulation. After $3 \mathrm{~min}$ network load of all protocols is almost same.

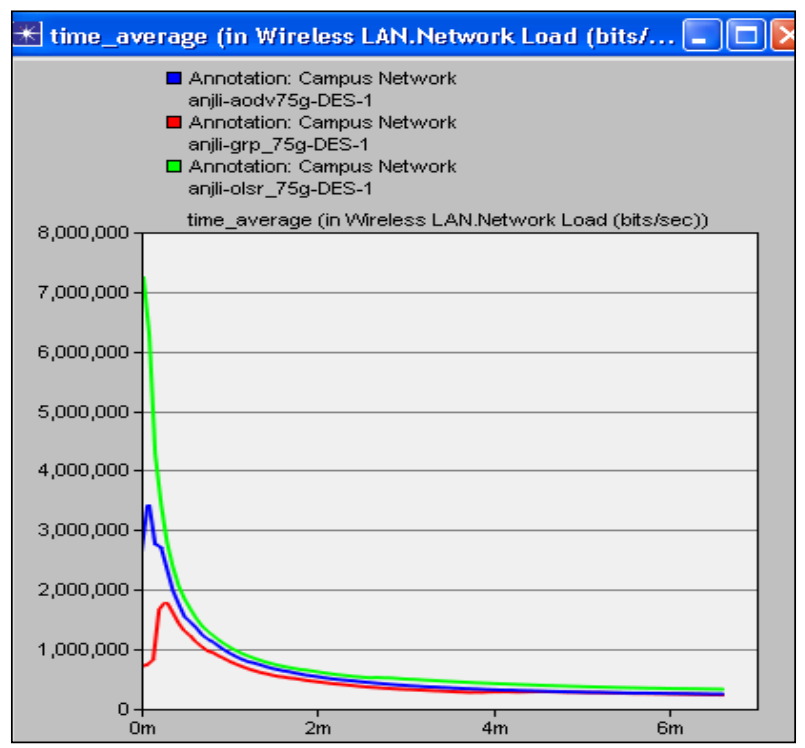

Fig. 5: Network Load for 802.11g with 75 nodes

From the Fig. 5 we conclude that in the starting, Network Load of OLSR is greatest but at the end $n / w$ load shows almost the same behavior with small fluctuations for all the three protocols. 


\subsubsection{Throughput}

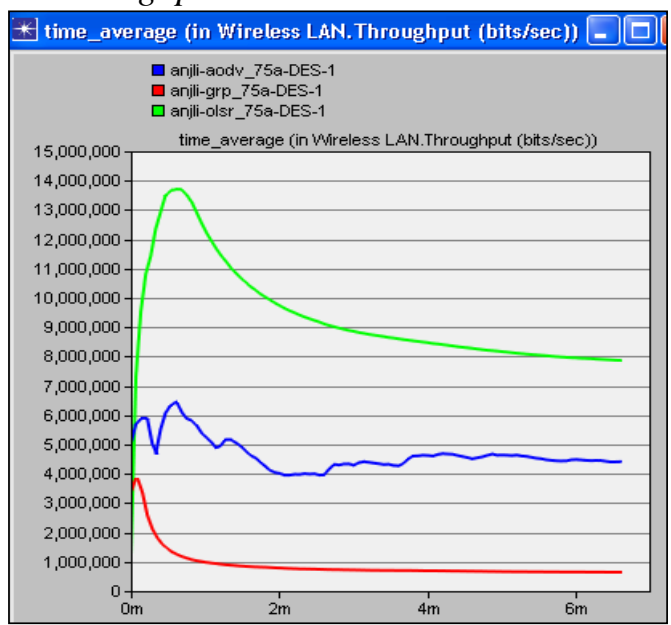

Fig. 6: Throughput for 802.11a with 75 nodes

Fig. 6 shows that throughput in OLSR is the highest and in GRP, we have the minimum throughput

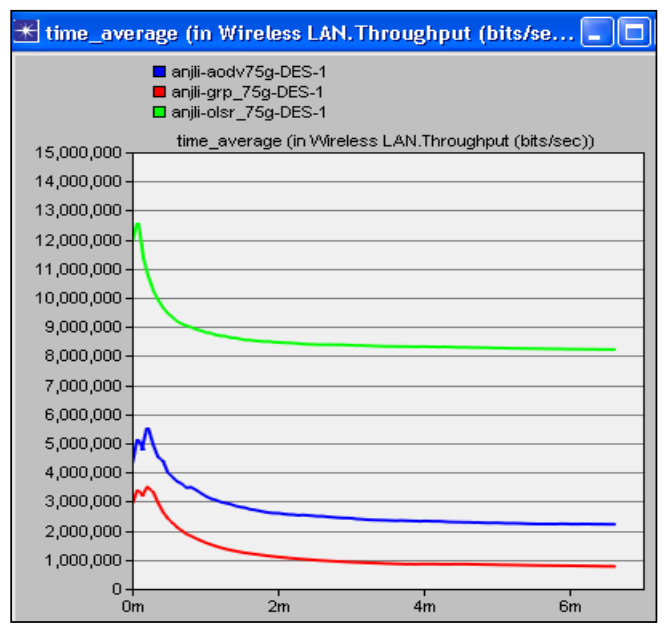

Fig. 7: Throughput for $802.11 g$ with 75 nodes

From Fig. 7 we find out that the throughput of OLSR is highest and in GRP we have the minimum throughput.

\subsubsection{Retransmission Attempts}

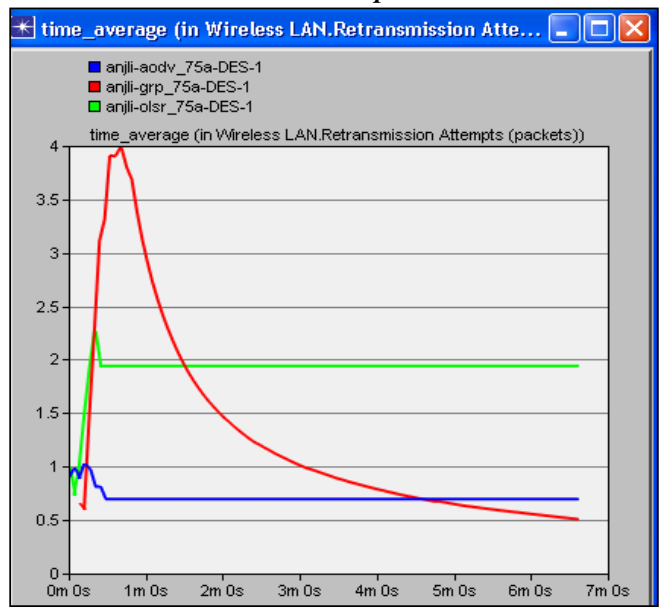

Fig. 8: Retransmission Attempts for 802.11a with 75 nodes
In Fig. 8, we see that GRP has more retransmission attempts than other two protocols in the starting. After $4.5 \mathrm{~min}$ we can conclude that retransmission attempts in OLSR are the highest and in GRP we have the minimum retransmission attempts.

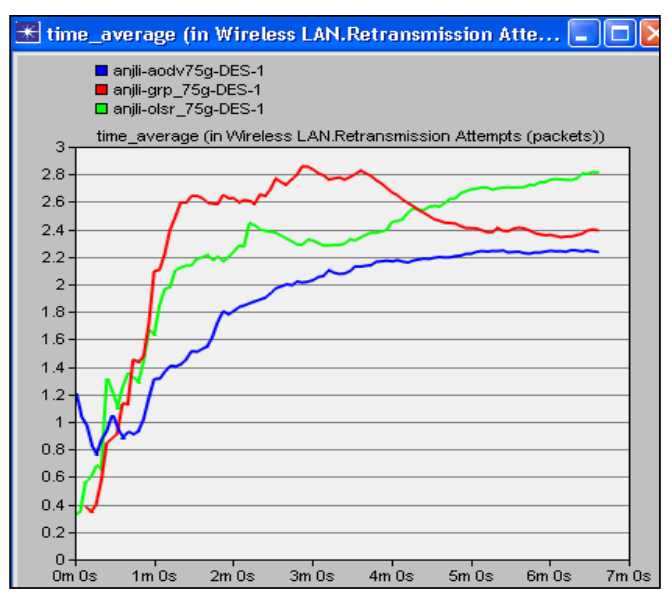

Fig. 9: Retransmission Attempts for 802.11 g with 75 nodes

According to simulation result in Fig. 9, we can see that OLSR has the maximum and AODV has minimum retransmission attempts.

We conclude from the above discussion that for 802.11a WLAN Standard with 75 nodes OLSR protocol is better than AODV and GRP in terms of media access delay and throughput. But retransmission attempts of GRP are less than other than other two protocols and network load of all the three protocols is almost same and for 802.11g WLAN Standard with 75 nodes it can be concluded that OLSR protocol is better than AODV and GRP in terms of media access delay and throughput. But retransmission attempts of AODV are less than other than other two protocols and network load of all the three protocols is almost same for 802.11g WLAN Standard with 75 nodes.

\subsection{AODV, OLSR and GRP in 150 nodes for 802.11a and $\mathrm{g}$ WLAN Standard}

The below figures (10 - 17) showing media access delay, network load, retransmission attempts and throughput in 150 mobile nodes scenario for 802.11a and g standard at $48 \mathrm{Mbps}$ data rate with AODV, GRP and OLSR respectively.

\subsubsection{Media Access Delay}

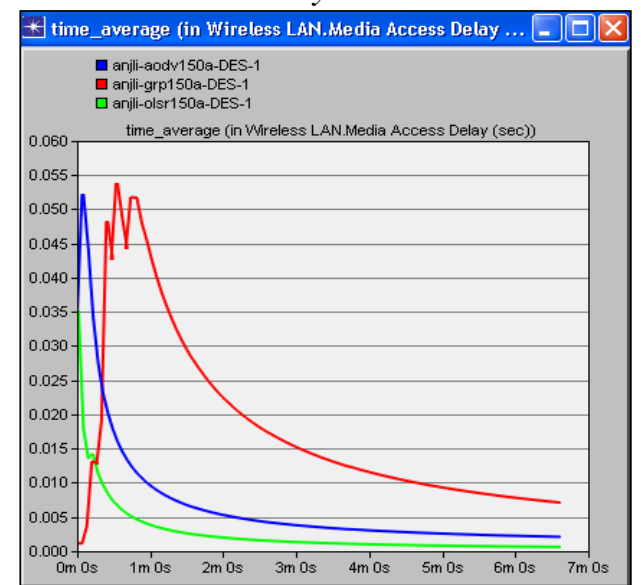

Fig. 10: Media Access Delay for 802.11a with 150 nodes 
Fig 10 depicts that media access delay of GRP is maximum and in OLSR we have minimum value of media access delay.

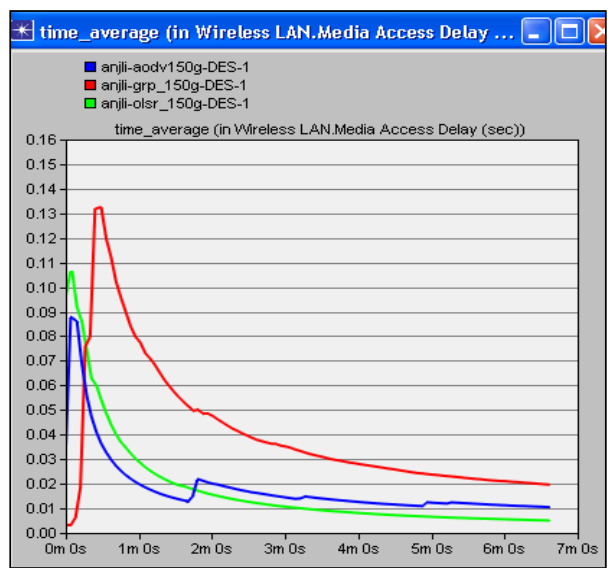

Fig 11: Media Access Delay for $802.11 \mathrm{~g}$ with 150 nodes

In Fig. 11 we find out that that media access delay in GRP is the highest and in OLSR we have the minimum media access delay.

\subsubsection{Network Load}

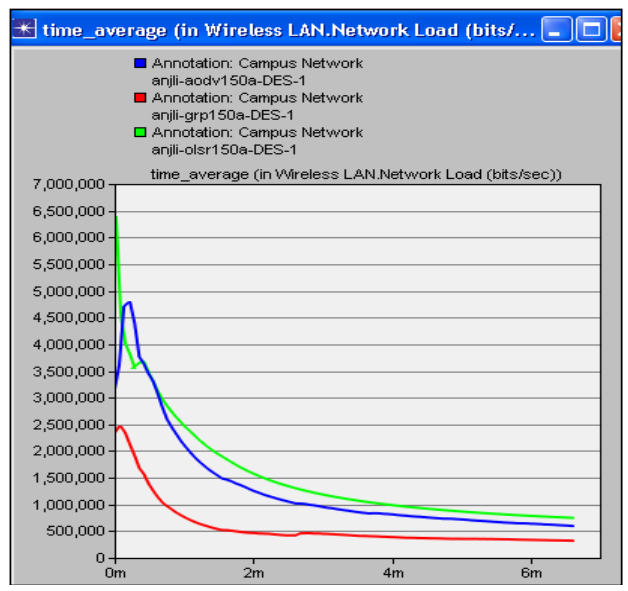

Fig. 12: Network Load for 802.11a with 150 nodes

According to simulation result in Fig. 12, we can see that OLSR has the maximum network load. We can order the network load respectively: OLSR > AODV > GRP.

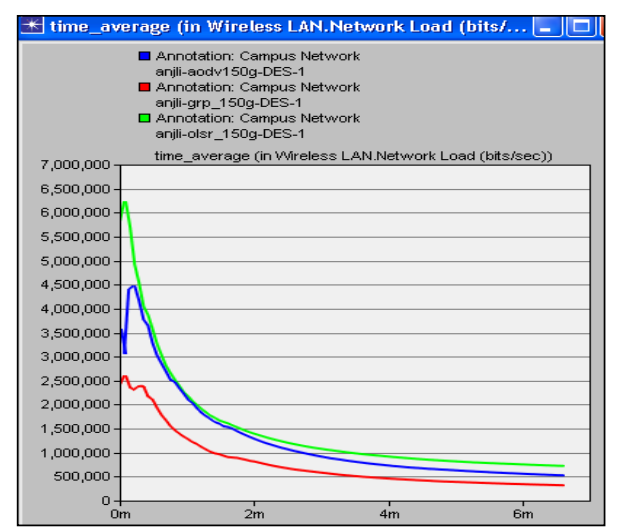

Fig.13: Network Load for $802.11 \mathrm{~g}$ with 150 nodes
According to simulation result in Fig. 13, we can see that OLSR has the maximum and GRP has minimum Network Load. We can order the network load respectively: OLSR $>$ AODV $>$ GRP.

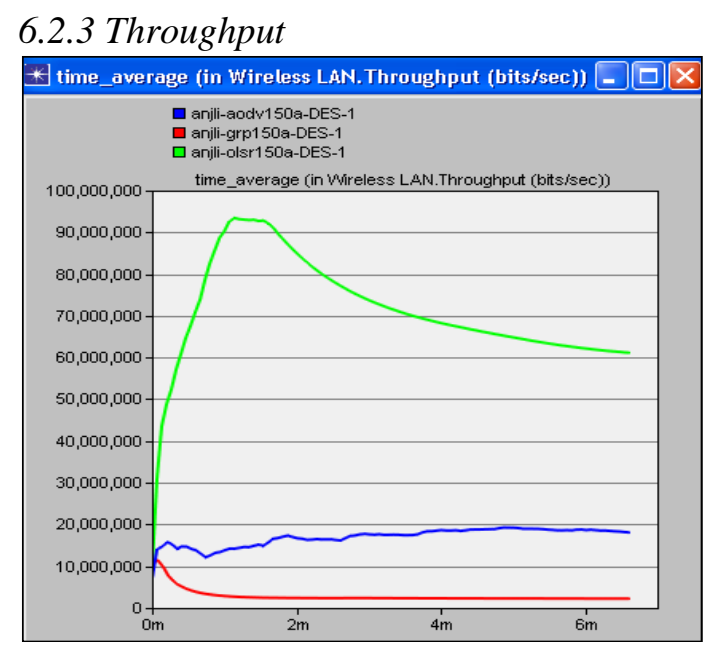

Fig. 14: Throughput for 802.11 a with 150 nodes

Fig. 14 shows that throughput in OLSR is the highest and in GRP, we have the minimum throughput.

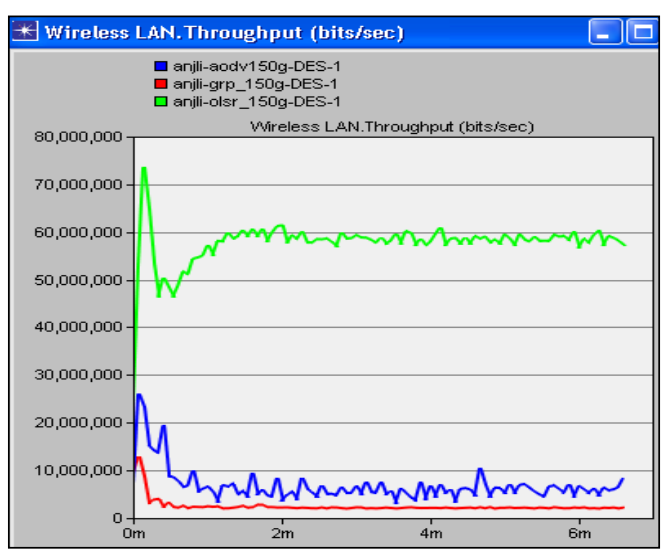

Fig. 15: Throughput for $802.11 g$ with 150 nodes

From Fig. 15 we can see that throughput in OLSR is the highest and in GRP, we have the minimum throughput.

\subsubsection{Retransmission Attempts}

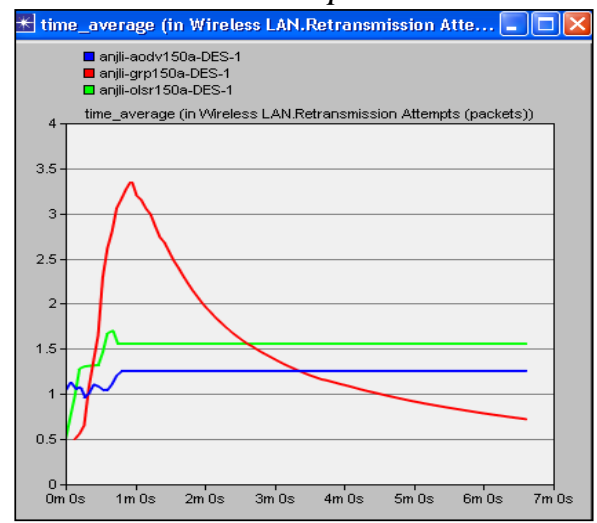

Fig. 16: Retransmission Attempts for 802.11a with 150 nodes 
In Fig. 16, we can conclude that retransmission attempts in OLSR are highest and in GRP we have the minimum retransmission attempts.

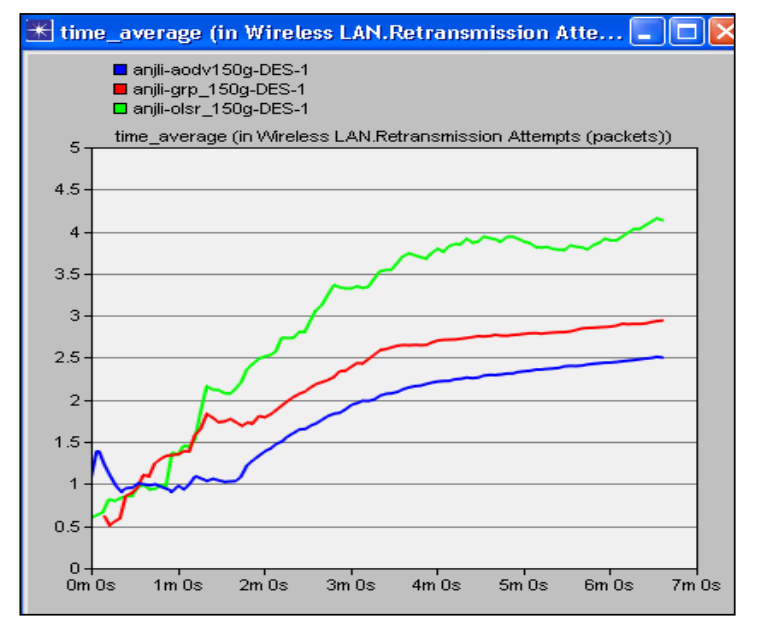

Fig. 17: Retransmission Attempts for $802.11 \mathrm{~g}$ with 150 nodes

Fig. 17 shows that retransmission attempts in OLSR are highest and in AODV, we have the minimum retransmission attempts.

We conclude from the above discussion that media access delay of OLSR is lesser and throughput of OLSR is higher than other two protocols. But retransmission attempts and network load of GRP are less than other two protocols. So we can conclude that OLSR protocol is better than other two protocols in terms of media access delay and throughput, but in terms of network load and retransmission attempts GRP is showing better results than AODV and OLSR protocol for 802.11a WLAN Standard with 150 nodes and for 802.11g WLAN Standard with 150 nodes it can be concluded that OLSR protocol is better than AODV and GRP in terms of media access delay and throughput. But network load of GRP protocol is minimum and in terms of retransmission attempts AODV protocol is showing better results than OLSR and GRP.

\section{CONCLUSION}

In this paper performance of Reactive (AODV), Hybrid (GRP) and Proactive protocol (OLSR) is evaluated for metrics like Media Access Delay, Network Load, Throughput and Retransmission Attempts by varying number of nodes and version of IEEE 802.11 WLAN Standard. From the above discussion we find out that OLSR performs best in each case in terms of media access delay and throughput. In terms of retransmission attempts GRP is showing better results than AODV and OLSR for 75 and 150 nodes with 802.11a technology but for $802.11 \mathrm{~g}$ technology AODV outperforms the two OLSR and GRP. In 75 mobile nodes network load of all the three protocols is almost same for both the technologies.GRP offer good results in offering low load on the network than OLSR and GRP respectively in case of 150 nodes for both technologies i.e. 802.11a and g. We summarized the results in table 6 and 7 .
TABLE 6 Resultant Values for 802.11a WLAN Standard

\begin{tabular}{|l|l|l|l|l|}
\hline Nodes & $\begin{array}{l}\text { Performance } \\
\text { Metrics }\end{array}$ & AODV & OLSR & GRP \\
\hline 75 & $\begin{array}{l}\text { Media Access } \\
\text { Delay }\end{array}$ & Medium & Low & High \\
\hline 75 & Network Load & High & Medium & Low \\
\hline 75 & Throughput & Medium & High & Low \\
\hline 75 & $\begin{array}{l}\text { Retransmission } \\
\text { Attempts }\end{array}$ & Medium & High & Low \\
\hline 150 & $\begin{array}{l}\text { Media Access } \\
\text { Delay }\end{array}$ & Medium & Low & High \\
\hline 150 & Network Load & Medium & High & Low \\
\hline 150 & $\begin{array}{l}\text { Retransmission } \\
\text { Attempts }\end{array}$ & Medium & High & Low \\
\hline
\end{tabular}

TABLE 7 Resultant Values for 802.11g WLAN Standard

\begin{tabular}{|c|c|c|c|c|}
\hline Nodes & $\begin{array}{c}\text { Performance } \\
\text { Metrics }\end{array}$ & AODV & OLSR & GRP \\
\hline 75 & $\begin{array}{c}\text { Media Access } \\
\text { Delay }\end{array}$ & Medium & Low & High \\
\hline 75 & Network Load & Same & Same & Same \\
\hline 75 & Throughput & Medium & High & Low \\
\hline Nodes & $\begin{array}{c}\text { Performance } \\
\text { Metrics }\end{array}$ & AODV & OLSR & GRP \\
\hline 150 & $\begin{array}{c}\text { Retransmission } \\
\text { Attempts } \\
\text { Dedia Access }\end{array}$ & Mow & High & Medium \\
\hline 150 & $\begin{array}{c}\text { Network Load } \\
\text { Attempts }\end{array}$ & Medium & High & Low \\
\hline 150 & $\begin{array}{c}\text { Retransmission } \\
\text { Ahroughput }\end{array}$ & Medium & High & Low \\
\hline
\end{tabular}


Therefore, we can conclude that under different environments, every protocol behaves differently because there are many parameters which differ under varied situations. From the above discussions we find out that OLSR outperforms the other two protocols in each scenario in terms of media access delay and throughput. So we can say that OLSR is better in MANET according to our simulation results but it is not necessary that OLSR always perform better in all the networks, its performance may vary by varying the network. At the end we came to the point from our simulation and analytical study that the performance of routing protocols vary with network and selection of accurate routing protocols according to the network, ultimately influence the efficiency of that network in magnificent way.

\section{ACKNOWLEDGEMENT}

Special thanks to PUNJABI UNIVERSITY, PATIALA and my supervisor Dr. Maninder Singh for providing continuous support throughout this work.

\section{REFERNCES}

[1] Pravin GhoseKar, Girish Katkar and Dr. Pradip Ghorpade, "Mobile Ad Hoc Networking: Imperatives and Challenges",IJCA, pp. 153-158, 2010.

[2] Razan Al-Ani, "Simulation and Performance Analysis Evaluation for Variant MANET Routing Protocols", International Journal of Advancements in Computing Technology, Volume 3, Number 1, Feb. 2011.

[3] Xiaoyan Hong, Kaixin Xu and Mario Gerla, "Scalable Routing Protocols for Mobile Ad Hoc Networks", IEEE Network, vol. 16, issue 4, pp 11-21, Aug.2002.

[4] Ravinder Ahuja, "Simulation based Performance Evaluation and Comparison of Reactive, Proactive and Hybrid Routing Protocols based on Random Waypoint Mobility Model", International Journal of Computer Applications, Vol. 7, No.11, pp. 20-24, Oct. 2010.
[5] Kuldeep Vats, Monika Sachdeva and Dr. Krishan Saluja, "Simulation and performance Analysis of OLSR, GRP, DSR Routing Protocol using OPNET", International Journal of Emerging trends in Engineering and Development, Vol.2 ,Issue 2, pp 390-404, March-2012.

[6] T.H. Clausen, "The optimized link state routing protocol evaluating through experiments and simulation" mind pass centre for distributed system,Aalborg university ,Denmark.

[7] Kuldeep Vats, "Simulation and performance analysis of OLSR routing protocol using OPNET" IJARCSSE ,Vol. 2, Issue 2, Feb. 2012.

[8] Mobile Ad Hoc Networking Working Group - AODV, http://www.ietf.org/rfc/rfc3561.txt.

[9] Naveen Bilandi, Harsh K Verma and Suryakant, “ Comparative Analysis of GRP and TORA MANET Routing Protocols using OPNET", Vol. 2, Issue 4, April 2012.

[10] C. Liu, J. Kaiser, “ A Survey of Mobile Ad Hoc network Routing Protocols", Network IEEE, Vol. 6, 2009, pp. 136 .

[11] N. Surayati, M. Usop, A. Abdullah,"Performance Evaluation of AODV, DSDV \& DSR Routing Protocol in Grid Environment", IJCSNS International Journal of Computer Science and Network Security, vol. 9, No.7, Jul. 2009, pp. 191-196.

[12] Z. Ghaleb, A. Mekhlafi and R. Hassan," Evaluation Study on Routing Information on Protocol and Dynamic Source Routing in Ad-Hoc Network", International IT in Asia Conference, 2011.

[13] Z. Ismail and R. Hassan, "Evaluation of Ad Hoc on Demand Distance Vector, Routing Protocol in HetMAN Architecture," Journal of Computer Science, vol. 6, Aug. 2010, pp. 830-836. 